Claremont Colleges

Scholarship@ Claremont

All HMC Faculty Publications and Research

HMC Faculty Scholarship

12-1-2009

\title{
Self-Organized Criticality in Sheared Suspensions
}

L. Corté

New York University

Sharon J. Gerbode

Harvey Mudd College

W. Man

New York University

D.J. Pine

New York University

\section{Recommended Citation}

L. Corte, S. J. Gerbode, W. Man, and D. J. Pine, “Self-organized criticality in sheared suspensions” Phys. Rev. Lett. 103, 248301 (2009). doi: 10.1103/PhysRevLett.103.248301

This Article is brought to you for free and open access by the HMC Faculty Scholarship at Scholarship @ Claremont. It has been accepted for inclusion in All HMC Faculty Publications and Research by an authorized administrator of Scholarship @ Claremont. For more information, please contact scholarship@cuc.claremont.edu. 


\title{
Self-Organized Criticality in Sheared Suspensions
}

\author{
L. Corté, ${ }^{1,2}$ S. J. Gerbode, ${ }^{3}$ W. Man, ${ }^{1,4}$ and D. J. Pine ${ }^{1}$ \\ ${ }^{1}$ Center for Soft Matter Research, New York University, 4 Washington Place, New York, New York 10003, USA \\ ${ }^{2}$ Mines-ParisTech, Centre des Matériaux, CNRS UMR7633, BP87 91003 Evry Cedex, France \\ ${ }^{3}$ Department of Physics, Cornell University, Ithaca, New York 14853, USA \\ ${ }^{4}$ Department of Physics and Astronomy, San Francisco State University, San Francisco, California 94132, USA
}

(Received 24 September 2009; published 7 December 2009)

\begin{abstract}
Recent studies reveal that suspensions of neutrally buoyant non-Brownian particles driven by slow periodic shear can undergo a dynamical phase transition between a fluctuating irreversible steady state and an absorbing reversible state. Using a computer model, we show that such systems exhibit self-organized criticality when a finite particle sedimentation velocity $v_{s}$ is introduced. Under periodic shear, these systems evolve, without external intervention, towards the shear-dependent critical concentration $\phi_{c}$ as $v_{s}$ is reduced. This state is characterized by power-law distributions in the lifetime and size of fluctuating clusters. Experiments exhibit similar behavior and, as $v_{s}$ is reduced, yield steady-state values of $\phi$ that tend towards the $\phi_{c}$ corresponding to the applied shear.
\end{abstract}

DOI: 10.1103/PhysRevLett.103.248301

A system is said to exhibit self-organized criticality (SOC) if it approaches a critical state with diverging time and length scales, under some small external but unadjusted drive [1,2]. SOC was first introduced by Bak, Tang and Wiesenfeld [3] as an explanation for the power-law behavior observed in some natural processes such as $1 / f$ noise or avalanches in sandpiles. Since then, many theoretical and numerical models have been shown to exhibit SOC $[2,4]$ but few physical realizations have been identified [2,5-10]. An interesting path to SOC has been explained for systems exhibiting a nonequilibrium phase transition between fluctuating "active" steady states and nonfluctuating "absorbing" states from which the system cannot escape [2]. In this Letter, we use such an absorbingstate phase transition and show through simulations and experiments that slowly sedimenting suspensions of particles subject to oscillatory shear represent a new class of self-organized critical systems.

Our approach is motivated by recent studies showing that suspensions of non-Brownian, neutrally buoyant particles can undergo a dynamical phase transition from absorbing to active states when they are slowly sheared back and forth $[11,12]$. For a given particle volume fraction $\phi$, two regimes are obtained depending on the strain amplitude $\gamma_{0}$ of the shear cycles. At low $\gamma_{0}$, the system organizes into an absorbing state where most particles return to their initial positions at the end of each cycle. Above a critical amplitude $\gamma_{0}^{c}(\phi)$, a finite fraction of the particles fail to return to their initial positions. Instead, they follow diffusivelike trajectories [13], resulting in an irreversible and fluctuating steady state. This transition from absorbing to active states exhibits key features of dynamical critical phenomena, with the fraction of diffusing particles as the order parameter and the power-law divergence of the relaxation time as $\gamma_{0} \rightarrow$ $\gamma_{0}^{c}(\phi)$.
PACS numbers: 47.57.E-, 05.70.Ln, 45.50.-j, 82.70.Kj

In new experiments, we explore the behavior of this same system in the presence of an external field, gravity, that couples to the suspended particles (see Fig. 1). The system is prepared with particles slightly denser than the suspending liquid. In the absence of shear, the particles sediment to form a dense random packing at the bottom of the cell. When the system is sheared, particles interact and diffuse upward until the shear-induced diffusion is balanced by the downward sedimentation caused by gravity in a process known as viscous resuspension [14].

Interestingly, we find in the current study that for oscillatory shear at an imposed strain amplitude $\gamma_{0}$, slowly sedimenting suspensions reach steady states where the volume fraction is very uniform. In the limit where sedimentation vanishes, the volume fraction of the suspension bed approaches the critical value $\phi_{c}\left(\gamma_{0}\right)$ delimiting active from absorbing steady states as measured in neutrally buoyant suspensions. To gain insight into the phenomenon,
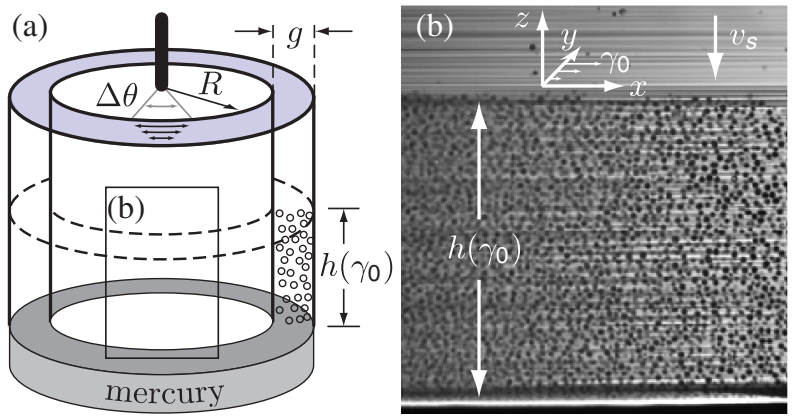

FIG. 1 (color online). (a) Setup for viscous resuspension under oscillatory shear in a Couette geometry. For a strain amplitude $\gamma_{0}=R \Delta \theta / 2 g$, the suspension bed rises to a height $h\left(\gamma_{0}\right)$. (b) The picture shows particles located in the plane of a laser sheet shined tangentially through the gap of a transparent Couette cell. Horizontal stripes are artifacts caused by imperfect matching of particle and fluid refractive indices. 
we consider a simple model based on shear-induced diffusion that mimics the competition between diffusion and sedimentation in the experimental system.

The model is based on a 2D model previously shown to capture the critical behavior of neutrally buoyant suspensions [12]. Here, we modify the model to account for sedimentation. $N$ point particles are randomly distributed in a rectangular box of width $L$ and height $H$. We define the horizontal $x$ axis as the shear flow direction and the vertical $y$ axis as the sedimentation direction as depicted in Fig. 2(a). Boundary conditions are periodic along the $x$ direction. The bottom of the box at $y=0$ is a hard impenetrable wall. An interaction distance $d$ is introduced to define collisions; it also sets the unit of length and is used as an effective particle diameter.

When the system is sheared back and forth with a strain amplitude $\gamma_{0}$, some particle pairs move closer than the interaction distance $d$. For each of these encounters, the colliding "active" particles are given a random displacement from their initial positions to simulate the irreversibility of their trajectories. The net displacement of a particle after one cycle is zero if it does not encounter any other particle during the cycle. The direction of the random displacements is uniformly distributed in the plane and the amplitude is uniformly distributed between 0 and a maximum value $\epsilon=0.5 d$ for the following results.

Sedimentation is introduced in the model by displacing all the particles downward by the same amount after each shear cycle. This defines a sedimentation velocity $v_{s}$ in units of $d$ per cycle. The unit time is one cycle and the shear rate is given by $\dot{\gamma}=\gamma_{0}$ per cycle.

Active particles are created in two ways: (i) an active particle activates an inactive particle by approaching it to
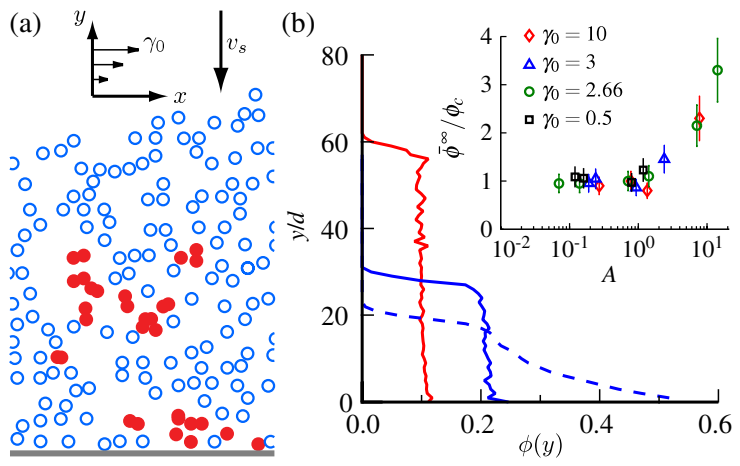

FIG. 2 (color online). Simulation: (a) Detail of the particle distribution in steady state. Particles are represented by circles of diameter $d$, the interaction distance. Filled symbols represent particles that will collide and diffuse irreversibly in the next shear cycle. (b) Area fraction profiles in steady state. Full lines show profiles in the $A<1$ regime for two strain amplitudes $\gamma_{0}=$ $10, A=0.78$ (blue or dark gray) and $\gamma_{0}=3, A=0.24$ (red or gray) ( $v_{s}=10^{-4} d /$ cycle). The dashed line shows the profile in the $A>1$ regime for $\gamma_{0}=3, A=2.4\left(v_{s}=10^{-3} d /\right.$ cycle $)$. Profiles are averaged over $2 \times 10^{4}$ shear cycles. Inset: $\bar{\phi}^{\infty} / \phi_{c}$ as a function of $A$ for several data sets: $\gamma_{0}=0.5,2.66,3$, and 10; $4.5<\kappa<20 d^{-1} ; 5 \times 10^{-5}<v_{s}<10^{-2} d /$ cycle. within less than $d$; (ii) an inactive particle moves downward due to sedimentation and collides another inactive particle that is pressed against the bottom wall, thus creating two new active particles. Furthermore, active particles can, through their random displacements, self-organize into a reversible configuration and become inactive [12]. If the suspension is organized into a fully reversible state with no active particles, activation can only occur through mechanism (ii). One recognizes here a process analogous to that of grain addition in sandpile models, which have been used to describe SOC [3].

Simulations with $10^{3}$ particles are run for $10^{5}$ shear cycles. After each cycle, we calculate the area fraction profile along the sedimentation axis $\phi(y) \equiv \frac{1}{L} \times$ $\int_{0}^{L} n(x, y) d x$ where $n(x, y)$ is the number of particles within a distance $d / 2$ from position $(x, y)$. The height of the suspension bed $h$ is taken to be the greatest $y$ for which $\phi(y) \neq 0$. Because the number of particles stacked per unit width $\kappa=N / L$ is conserved, $h$ is related at anytime to the mean area fraction of the suspension $\bar{\phi}$ by $h \bar{\phi}=\pi d^{2} \kappa / 4$. In the following, $\kappa=7.64 d^{-1}$ unless otherwise stated.

Steady state is achieved when the height fluctuates about a constant mean value $h^{\infty}$, i.e., constant mean area fraction $\bar{\phi}^{\infty}=\pi d^{2} \kappa / 4 h^{\infty}$. The system reaches the same steady state regardless of the initial particle distribution.

For a fixed $\gamma_{0}$, we observe two different steady-state regimes depending on the relative importance of sedimentation and shear-induced diffusion. The first regime resembles what is obtained in conventional viscous resuspension [14] and corresponds to the highest sedimentation velocities. Sedimentation and shear-induced diffusion compete such that an exponential density profile is established near the bottom, as shown by the dashed line in Fig. 2(b). The system is far from an absorbing state with most of the particles constantly colliding.

The second regime is obtained as $v_{s}$ is reduced towards zero: in steady state, clusters of active particles nucleate at the bottom wall and diffuse until the activity dies when the particles reorganize into a new reversible configuration. Figure 2(a) shows a snapshot of such a steady state with active particles close to the bottom wall and others at a finite height after activity has diffused away from the bottom. Particle density is nearly uniform in this regime, as illustrated by the full line profiles in Fig. 2(b).

This second regime is the one of interest for SOC. In this case, for a given $\gamma_{0}, \bar{\phi}^{\infty}$ fluctuates around a value that is near the critical concentration $\phi_{c}\left(\gamma_{0}\right)$ delimiting the absorbing and active steady states for the case without sedimentation. The transition between the regime with heightdependent profiles and the regime with nearly uniform profiles can be characterized by a dimensionless number $A \equiv \tau_{D} / \tau_{s}$ expressing the ratio of a characteristic time for shear-induced diffusion $\tau_{D}$ to a characteristic time for sedimentation $\tau_{s}$.

The uniform profile, if it is reached, is obtained near the critical point, meaning that $\bar{\phi}^{\infty} \approx \phi_{c}\left(\gamma_{0}\right)$ and 
$h^{\infty} \approx \pi d^{2} \kappa / 4 \phi_{c}\left(\gamma_{0}\right)$. In the absence of diffusion, the area fraction at the bottom wall would increase due to sedimentation from $\phi_{c}$ to $2 \phi_{c}$ in a time $\tau_{s}=\xi / v_{s}$, where $\xi=$ $\sqrt{\pi d^{2} / 4 \phi_{c}}$ is the mean interparticle spacing. Shearinduced diffusion redistributes this increase in $\phi$ throughout the whole suspension height $h^{\infty}$ in a time $\tau_{D}=$ $h^{\infty 2} / 4 D$, where $D$ is the diffusivity under strain amplitude $\gamma_{0}$ and at area fraction $2 \phi_{c}$. The ratio of these two times is given by the dimensionless number

$$
A=\frac{\tau_{D}}{\tau_{s}}=\left(\frac{\pi}{\phi_{c}}\right)^{3 / 2} \frac{d^{3} \kappa^{2} v_{s}}{4 D} .
$$

As expected, $A$ goes to zero when gravity vanishes. It describes the transition between the two steady-state regimes remarkably well [see Fig. 2(b) inset]. All data collapse onto a single master curve. For $A>1$, shear-induced diffusion cannot homogenize the suspension. The area fraction varies strongly along the sedimentation axis and $\bar{\phi}^{\infty}>\phi_{c}$. For $A<1$, transport is dominated by shearinduced diffusion. The system finds steady states with sharp profiles and a mean area fraction that fluctuates around the critical value $\phi_{c}\left(\gamma_{0}\right)$.

To study the criticality of the steady states in the $A<1$ regime, we measure the probability densities $P(s)$ and $P(t)$ of the size and lifetime of active clusters. An active cluster is defined in both space and time as the sequence of colliding particles generated by one nucleation event through process (ii). Figure 3(a) shows the shape in space-time coordinates for one cluster. Its lifetime $t$ is the
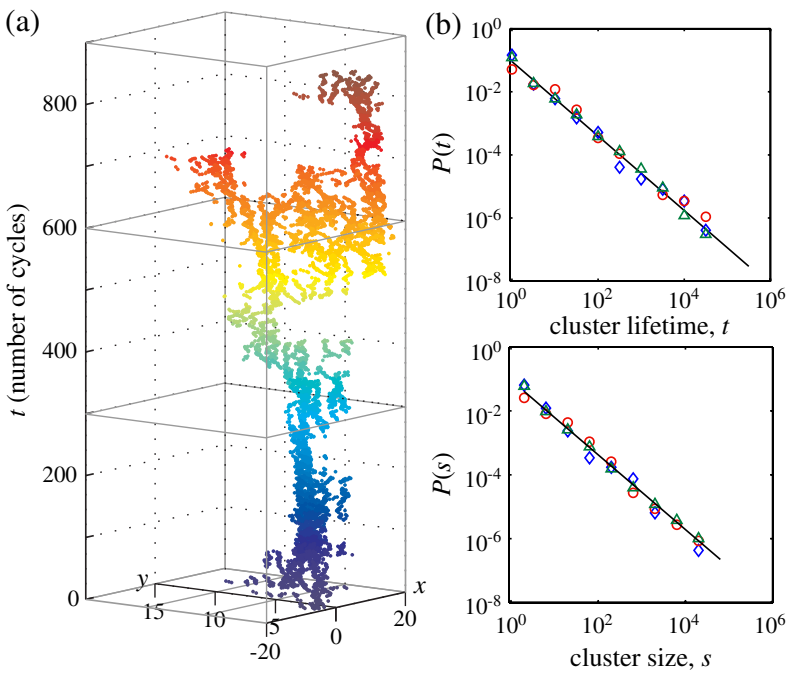

FIG. 3 (color online). Simulation: (a) Particles forming an active cluster in space-time coordinates. Symbols represent particle centers with their color changing with time from cluster nucleation. The cluster nucleates at the bottom wall $(x, y, t) \approx$ $(-4,0,0)$ and lasts nearly 900 cycles. (b) Distributions of cluster lifetime (top) and size (bottom) for three different strain amplitudes $\gamma_{0}=10, A=0.78(\diamond) ; \gamma_{0}=3, A=0.24(\bigcirc)$, and $\gamma_{0}=$ $0.5, A=0.12(\triangle)\left(v_{s}=10^{-4} d \times\right.$ cycle $\left.^{-1}\right) .200$ to 600 clusters are recorded over $9 \times 10^{4}$ cycles. Lines show power-law fits $P(t) \sim t^{-1.20}$ and $P(s) \sim s^{-1.22}$ number of cycles from its birth to its death, about 900 cycles in this example. Its size $s$ is determined by summing the number of active particles in the cluster at each cycle over its entire life.

Figure 3(b) shows $P(s)$ and $P(t)$ for three values of $\gamma_{0}$. These distributions follow a remarkable power law over four decades. Fitting these data gives $P(s) \sim s^{-\tau}$ with $\tau=$ $1.22 \pm 0.05$ and $P(t) \sim t^{-\alpha}$ with $\alpha=1.20 \pm 0.05$. Such power-law distributions are the signature of critical behavior. They confirm that the model system exhibits SOC as it is able to maintain itself near its critical point without any special tuning but a vanishing external drive, which in this case is sedimentation.

The case of real non-Brownian suspensions is studied with the experiment depicted in Fig. 1(a). The suspension consists of polymethylmethacrylate (PMMA) particles of diameter $d=230 \pm 20 \mu \mathrm{m}$ suspended in a viscous liquid. The suspending liquid is a mixture that is commonly used to match both the density and refractive index of PMMA [15]. Its composition is slightly altered to create a density mismatch and induce particle sedimentation. In this liquid, a lone tracer particle sediments at about $0.006 \pm$ $0.002 \mu \mathrm{m} / \mathrm{s}$ at $20^{\circ} \mathrm{C}$. Fluorescent dye is also added to the fluid so that particles appear as dark spots in the plane of a sheet of laser light 200-300 $\mu \mathrm{m}$ thick, as shown in Fig. 1(b). The Couette cell and imaging system are described elsewhere [11,12].

To start, the suspension is sedimented in a random packing resting on a layer of mercury at the bottom of the cell. An oscillatory shear is then applied by rotating the inner cylinder back and forth about its axis. The strain amplitude $\gamma_{0}$ of these oscillations starts at 0.2 and increases in steps up to 5.0. Approximately 1500 shear cycles for each $\gamma_{0}$ step are necessary to reach steady state. Two series of experiments are performed for oscillation periods of 5 and $25 \mathrm{~s}$, which gives a sedimentation velocity $v_{s}$ for a lone particle of about 0.03 and $0.15 \mu \mathrm{m} /$ cycle, respectively. In those conditions, the Reynolds number of the system [11] is kept less than $10^{-2}$, where the motion of pure fluids is reversible.

In steady state, the suspension has a very uniform volume fraction, as can be seen in Fig. 1(b). The heightdependent particle density is determined by counting the number of particles at a height $z$ in a portion of the laser sheet. Figure 4(a) shows the steady-state profiles $\rho^{\infty}(z)$ obtained for various strain amplitudes. For each $\gamma_{0}$, these profiles have a well-defined height $h^{\infty}\left(\gamma_{0}\right)$ and exhibit almost no dependence on $z$. The steady-state mean volume fraction can then be given by $\bar{\phi}^{\infty}\left(\gamma_{0}\right)=\phi_{0} h_{0} / h^{\infty}\left(\gamma_{0}\right)$ where $h_{0}$ and $\phi_{0}$ are the height and volume fraction of the fully sedimented suspension. Here, $h_{0}=5.0 \pm 0.3 \mathrm{~mm}$ and we choose $\phi_{0}=0.6 \pm 0.04$ based on other measurements with the error bars indicating the uncertainty.

Figure 4(b) shows $\bar{\phi}^{\infty}\left(\gamma_{0}\right)$ for the two values of $v_{s}$ as well as $\phi_{c}\left(\gamma_{0}\right)$ for the critical case of neutrally buoyant suspensions [11,12]. The critical values $\phi_{c}$ are determined separately by varying $\gamma_{0}$ in experiments with different $\phi$. 


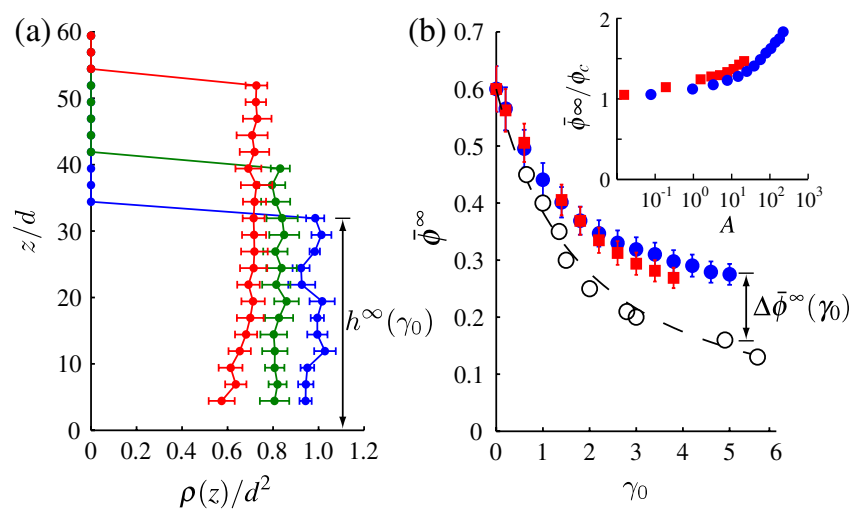

FIG. 4 (color online). Experiment: (a) Particle density profiles in steady state for $\gamma_{0}=2.8$ (left), 1.0 (middle), and 0.2 (right). Profiles are averaged over 50 shear cycles. Error bars correspond to standard deviations. (b) Steady-state volume fraction as a function of $\gamma_{0}$. Solid symbols show mean volume fraction $\bar{\phi}^{\infty}\left(\gamma_{0}\right)$ measured in the sedimenting case for two different oscillation periods ( $25 \mathrm{~s}, \mathbf{\square}: 5 \mathrm{~s})$. Open symbols $(\bigcirc)$ show the critical line $\phi_{c}\left(\gamma_{0}\right)$ as measured with neutrally buoyant suspensions. The line is a power-law fit scaling as $\left(1 / 0.6+\gamma_{0}\right)^{-1}$.

For $\gamma_{0}<1, \phi^{\infty}\left(\gamma_{0}\right)$ and $\phi_{c}\left(\gamma_{0}\right)$ are undistinguishable within the experimental error and the suspension is in a near critical steady state. For larger $\gamma_{0}$, however, the gap to criticality $\Delta \bar{\phi}^{\infty}\left(\gamma_{0}\right)=\bar{\phi}^{\infty}\left(\gamma_{0}\right)-\phi_{c}\left(\gamma_{0}\right)$ increases and suspensions are denser than in their critical state.

As for the 2D model, this transition to the critical steadystate regime is well described by the dimensionless ratio $A$. For the 3D geometry of the experiment,

$$
A=\left(\frac{\pi}{6 \phi_{c}}\right)^{5 / 6} \frac{d^{5} \kappa^{2} v_{s}}{4 D} .
$$

Here, $\kappa=6 h_{0} \phi_{0} / \pi d^{3}$ is the number of particles stacked per unit area in the $z$ direction.

In the real case, hydrodynamic interactions between particles induce some additional dependence on $\phi$. In particular, particle sedimentation within a sheared suspension is strongly hindered by interparticle interactions. A more realistic sedimentation velocity is given by $\tilde{v}_{s}=$ $f\left(\phi_{c}\right) v_{s}$ where $f(\phi)$ is a decreasing function of $\phi$ that equals 1 for $\phi=0$. Here, we use the hindrance function defined in [14]. Measurements in neutrally buoyant suspensions also show that diffusivity along the $z$ axis scales as $D_{0} \phi^{2}\left(\phi / \phi_{c}-1\right)^{\beta}$ with $D_{0} \approx 0.02 \pm 0.01 d^{2} /$ cycle and $\beta \approx 1.3 \pm 0.3$. Hence, we determine the diffusivity at $\phi=2 \phi_{c}$ using $\tilde{D}=4 \phi_{c}^{2} D_{0}$ where $\phi_{c}$ is estimated from the power-law fit shown in Fig. 4(b).

The inset in Fig. 4(b) shows $\bar{\phi}^{\infty} / \phi_{c}$ as a function of $A$ calculated with $\tilde{v}_{s}$ and $\tilde{D}$. As for the model, a transition is observed around $A=1$ and the mean volume fraction approaches the critical value as the densification due to gravity is suppressed by shear-induced diffusion through the suspension height $(A \rightarrow 0)$. For increasing $\gamma_{0}, \phi_{c}$ goes to 0 scaling as $1 / \gamma_{0}$ and $f\left(\phi_{c}\right) \simeq 1$. As a result, $A$ increases as $1 / \phi_{c}^{17 / 6}$, much more rapidly than in the $2 \mathrm{D}$ model. This suggests that SOC states are more easily attained experimentally for small strain amplitudes, consistent with the data presented in Fig. 4(b), and for suspension beds with few stacked particles $(\kappa \ll 1)$.

Perhaps the clearest previous observation of SOC is that of Moeur et al. [7], who observed SOC in liquid ${ }^{4} \mathrm{He}$ near the equilibrium superfluid transition. By contrast, here SOC is observed near a nonequilibrium transition. In early models of SOC involving nonequilibrium avalanches, the origin of the criticality was not always clear. Here, the criticality comes from a nonequilibrium phase transition recently identified by Corté $e$ t al. [12], who suggested that the transition was an absorbing phase transition, possibly related to conserved directed percolation [16]. It may be that other systems exhibiting SOC have the same type of criticality observed in this system. Indeed, this is what has been suggested by Dickman et al. [1,2] for sandpile models such as the Manna model.

We thank Paul Chaikin for illuminating discussions. This work was supported partially by the MRSEC Program (NSF Grant No. DMR-0820341). L. C. received support from a Lavoisier Fellowship from the French government and S. J. G. from Cornell's IGERT Program in Nonlinear Systems (NSF Grant No. DGE-9870631).

[1] R. Dickman, A. Vespignani, and S. Zapperi, Phys. Rev. E 57, 5095 (1998).

[2] R. Dickman, M. A. Munoz, A. Vespignani, and S. Zapperi, Braz. J. Phys. 30, 27 (2000).

[3] P. Bak, C. Tang, and K. Wiesenfeld, Phys. Rev. A 38, 364 (1988).

[4] D. Turcotte, Rep. Prog. Phys. 62, 1377 (1999).

[5] P. J. Cote and L. V. Meisel, Phys. Rev. Lett. 67, 1334 (1991).

[6] V. Frette, K. Christensen, A. Malthe-Sorenssen, J. Feder, T. Jossang, and P. Meakin, Nature (London) 379, 49 (1996).

[7] W. A. Moeur, P. K. Day, F. C. Liu, S. T. P. Boyd, M. J. Adriaans, and R. V. Duncan, Phys. Rev. Lett. 78, 2421 (1997).

[8] C. Aegerter, M. Welling, and R. Wijngaarden, Europhys. Lett. 65, 753 (2004).

[9] J. Strle, D. Vengust, and D. Mihailovic, Nano Lett. 9, 1091 (2009).

[10] J. Sprakel, E. Spruijt, M. A. C. Stuart, M. A. J. Michels, and J. van der Gucht, Phys. Rev. E 79, 056306 (2009).

[11] D. J. Pine, J. P. Gollub, J. F. Brady, and A. M. Leshansky, Nature (London) 438, 997 (2005).

[12] L. Corté, P. Chaikin, J. P. Gollub, and D. J. Pine, Nature Phys. 4, 420 (2008).

[13] E. C. Eckstein, D. G. Bailey, and A. H. Shapiro, J. Fluid Mech. 79, 191 (1977).

[14] A. Acrivos, R. Mauri, and X. Fan, Int. J. Multiphase Flow 19, 797 (1993).

[15] G. P. Krishnan, S. Beimfohr, and D. T. Leighton, J. Fluid Mech. 321, 371 (1996).

[16] G. I. Menon and S. Ramaswamy, Phys. Rev. E 79, 061108 (2009). 